CERN-EP/87-01

6 January 1987

\title{
ANTIPROTON-PROTON POTENTIAL FROM CROSS-SECTION DATA AT SMALL MOMENTA
}

\author{
T. -A. Shibata
}

Physikalisches Institut der Universität,

Heidelberg, Fed. Rep. Germany

\begin{abstract}
Antiproton-proton cross-sections for annihilation, elastic scattering, and the charge-exchange reaction between 180 and $600 \mathrm{MeV} / \mathrm{c}$ have been analysed with a potential model. It is shown that the cross-sections are reproduced with a model which consists of a one-pion-exchange potential and a short-range complex annihilation potential. The ranges of the interactions in these three reaction channels are investigated, and it is shown that annihilation takes place at short distances of less than $2 \mathrm{fm}$, while the charge-exchange reaction is sensitive to the region up to $4 \mathrm{fm}$.
\end{abstract}

(Sublnitted to Physics Letters B)

*) Visitor at CERN, Geneva, Switzerland. 


\section{INTRODUCTION}

In a recent experiment at the Low-Energy Antiproton Ring (LEAR) at CERN, a precise measurement has been performed of the antiproton-proton cross-sections for elastic scattering, the charge-exchange reaction, and annihilation in the beam momentum range between 180 and $600 \mathrm{MeV} / \mathrm{c}$. The real-to-imaginary ratio of the forward elastic-scattering amplitude [1], the partial-wave compositions of the elastic and inelastic cross-sections [2], and a model comparison of the differential charge-exchange cross-section [3], derived from these data, have been presented.

The major motivation of this experiment was to compare nucleon-nucleon (NN) and antinucleon-nucleon ( $\overline{\mathrm{N} N}$ ) interactions at small momenta, and in this way extract additional information on the mechanism of the NN interaction. The comparison is usually performed by using boson-exchange models for the NN interaction and applying the G-parity transformation for the $\bar{N} N$ system. In the $\bar{N} N$ interaction, the annihilation is taken into account by introducing an empirical annihilation potential [4-7], or by using a boundary condition model [8], or quark-rearrangement models [9-11].

In the present paper, however, we would like to show that the cross-section data so far available can be well reproduced by a model with a long-range onepion-exchange potential and a short-range complex annihilation potential. The complex potential is adjusted so as to simulate the $\overline{\mathrm{N}} \mathrm{N}$ annihilation and possible other contributions from short-range boson exchanges. We use a function of the woods-Saxon type for the complex potential. The use of this potential is based on our experience that the annihilation is determined by the depth of the potential, typically $100 \mathrm{MeV}$, at a distance of around $1 \mathrm{fm}$.

with this potential, we investigate ranges of the interactions in three reaction channels: the annihilation, the charge-exchange $(\bar{p} p \rightarrow \bar{n} n)$ and the elastic scattering one. The ranges of annihilation and of nuclear force were discussed elsewhere using an isoscalar potential [12]. In the present analysis, the arguments are extended so as to include the charge-exchange reaction. The charge-exchange reaction is determined by the difference between the $I=1$ and $I=0$ amplitudes, while the elastic scattering is determined by their sum. The simultaneous analysis of data on elastic scattering and the charge-exchange reaction thus provides an insight into the isospin dependence of the potential.

This analysis leads, consequently, to introducing one parameter characterizing the range of annihilation, the 'annihilation radius', which is around $1 \mathrm{fm}$. The one-pion-exchange potential (OPEP) and the value of the annihilation radius determine the major characteristics of pp cross-sections at small momenta. Other models, as we will show in the case of the Dover-Richard model, also give a good fit provided that they reproduce the proper value of the anninila- 
tion radius. At the end of the paper, we point out that the charge-exchange cross-section could be an important observable for detailed studies of the medium- and short-range interactions.

\section{POTENTIAL}

We solve the Schrödinger equation with the following potential:

$$
v=v_{0}+i w+v_{\text {OPEP }}
$$

where $v_{0}$ and $w$ are functions of the woods-Saxon type, and $v_{\text {OPEP }}$ is the G-parity-transformed OPEP:

$$
\begin{aligned}
& \mathrm{v}_{0}=-c_{0} /\left[1+\exp \left[\left(r-r_{0}\right) / a_{0}\right]\right], \\
& w=-c_{1} /\left[1+\exp \left[\left(r-r_{1}\right) / a_{1}\right]\right], \\
& v_{\text {OPEP }}=-\left(\tau_{1} \cdot \tau_{2}\right)\left[\left(\sigma_{1} \cdot \sigma_{2}\right) v_{S S}+S_{12} v_{T}\right] .
\end{aligned}
$$

$\mathrm{V}_{\mathrm{ss}}$ is a Yukawa function with the range parameter of the pion mass, $s_{12}$ the tensor operator, and $v_{T}$ a radial function for the tensor term. The $\pi N N$ coupling constant 14.43 is used in the OPEP. The OPEP is kept constant at distances of less than $0.8 \mathrm{fm}$ with the value at $0.8 \mathrm{fm}$.

Parameters in $v_{0}$ and $w$ are optimized to reproduce the cross-sections for annihilation, elastic scattering, and the charge-exchange reaction between 290 and $600 \mathrm{MeV} / \mathrm{c}$. The depth of the potential at distances less than $0.5 \mathrm{fm}$ does not affect the cross-sections since the wave function is damped there. The parameters in $v_{0}$ and $w$ are strongly correlated. We find a good solution keeping $c_{0}=c_{1}, r_{0}=r_{1}$, and $a_{0}=a_{1}: c_{0}=500 \mathrm{MeV}, r_{0}=0.74 \mathrm{fm}$, and $a_{0}=0.2 \mathrm{fm}$. Each part of the potential is shown in fig. 1 . Whether OPEP is attractive or repulsive depends on the states since the isospin product $\left(\tau_{1}{ }^{-} \tau_{2}\right)$ and the spin product $\left(\sigma_{1} \cdot \sigma_{2}\right)$ take 1 for triplet states and -3 for singlet states. The cross-sections are calculated from phase shifts obtained by solving the Schrödinger equation, using the formula given by stapp et al. [13], which includes the spin effect. The results of the calculations are compared with the experimental data $[2,3]$ in fig. 2 .

The isospin dependence of the elastic and charge-exchange cross-sections is expressed as:

$$
\mathrm{do}_{\mathrm{el}} / \mathrm{dQ}=|[\mathrm{f}(\mathrm{I}=1)+\mathrm{f}(\mathrm{I}=0)] / 2|^{2}
$$




$$
\mathrm{do}_{\mathrm{CEX}} / \mathrm{dQ}=|[\mathrm{f}(\mathrm{I}=1)-\mathrm{f}(\mathrm{I}=0)] / 2|^{2}
$$

where $f$ is the scattering amplitude of the $I=1$ or $I=0$ states. The isospin difference of the scattering amplitudes is shown by an arrow in fig. 3 in the Argand diagram for the ${ }^{3} \mathrm{p}_{1}$ wave at $290 \mathrm{MeV} / \mathrm{c}$. In the present model, OPEP is the only source of isospin dependence. If the OPEP is switched off, the isospin dependence vanishes and the phase shifts move to the dotted line in fig. 3 . The arrow is almost horizontal, indicating that the real parts of the scattering amplitudes determine the charge-exchange cross-section. Therefore, in a later section, we plot the real parts of the scattering amplitudes in order to investigate the radial dependence of the charge-exchange reaction.

\section{RANGE OF ANNIHILATION}

We have just shown that the potential model, which consists of the longrange OPEP and the short-range annihilation potential, can reproduce the crosssection data. Now we investigate the annihilation probability, particularly its radial dependence, and introduce a measure of the range of the annihilation, the 'annihilation radius'. The incident plane wave and the solution of the schrödinger equation, $\psi$, are expanded, respectively, as:

$$
\begin{aligned}
e^{i k z}= & {\left[(21+1) i^{1} j_{1}(k r) P_{1}(\cos \theta),\right.} \\
& \begin{aligned}
1 \\
\end{aligned} \quad=\left[(21+1) i^{1} R_{1}(r) P_{1}(\cos \theta),\right.
\end{aligned}
$$

where $j_{1}$ is the spherical Bessel function and $P_{1}$ is the Legendre polynomial.

The annihilation cross-section is expressed in terms of the imaginary potential $W$ and the wave function $\Psi[14]$ :

$$
\begin{aligned}
& \sigma_{\text {ann }}=-(2 / \hbar v) \int w|\Psi|^{2} d Q \\
& =-(8 \pi / \hbar v)\left(1 / k^{2}\right) \sum_{1}^{[}(21+1) \int w\left[\left[\left|R_{1}(I=1)\right|^{2}+\left|R_{1}(I=0)\right|^{2}\right] / 2\right](k r)^{2} d r ，
\end{aligned}
$$

where $v$ is the relative velocity, and $|\psi|^{2}$ is the average of the squared wave functions with $I=1$ and $I=0$.

Figure $4 a$ shows $W_{1} j_{1} \cdot(k r)$, and the real and imaginary parts of $R_{1} \cdot(k r)$ for ${ }^{13} \mathrm{P}_{1}$ at $290 \mathrm{MeV} / \mathrm{c}$, for example. The density probability $\left|R_{1}\right|^{2}(\mathrm{kr})^{\frac{1}{2}}$ and the annihilation probability $w\left|R_{1}\right|^{2} \cdot(k r)^{2}$ for ${ }^{13} p_{1}$ at $290 \mathrm{MeV} / \mathrm{c}$ are plotted in fig. $4 b$. We see that the annihilation takes place at distances of less than 
$2 \mathrm{fm}$. As a measure of the range of the annihilation, it seems reasonable to define the annihilation radius $r_{a}$ as follows:

$$
\int_{0}^{r_{a}} w|\psi|^{2} r^{2} d r=\int_{r_{a}}^{\infty} w|\psi|^{2} r^{2} d r .
$$

The $r_{a}$ is insensitive to local structures of the potential. The value of $r_{a}$ does not depend on the states very much, and ranges between 0.9 and $1.2 \mathrm{fm}$. It is interesting to note that at the distance where the attraction is strong in the NN potential, the $\bar{N} N$ potential is dominated by the annihilation.

\section{THE RANGES OF THE CHARGE-EXCHANGE REACTION AND OF ELASTIC SCATTERING}

In this section we investigate the ranges of the charge-exchange reaction and of elastic scattering by plotting the radial dependence of the scattering amplitudes. The scattering amplitude $f$ in eqs. (5) and (6) is expressed in terms of the potential $v, j_{1}$ and $R_{1}[14]$ :

$$
\begin{aligned}
f & =(1 / k) \underset{l}{[}(21+1) T_{1} P_{1}(\cos \theta), \\
T_{1} & =-\left(2 \mu / \hbar^{2}\right)(1 / k) \int j_{1}(k r) V(r) R_{1}(r) \cdot(k r)^{2} d r,
\end{aligned}
$$

where $\mu$ is the reduced mass or half the proton mass. Figure $4 \mathrm{c}$ shows the real part of $-j_{1} V R_{l} \cdot(k r)^{2}$ for ${ }^{33} \mathrm{p}_{1}$ and ${ }^{13} \mathrm{p}_{1}$ at $290 \mathrm{MeV} / \mathrm{c}$. The $j_{1}$ is real while $R_{1}$ is complex. Therefore, $T_{1}$ is determined by the combination of the complex potential $\mathrm{V}$ and the complex wave function $\mathrm{R}_{1}$. The oscillation seen in ${ }^{33} \mathrm{P}_{1}$ below $2 \mathrm{fm}$ is due to the node of $R_{1}$.

The charge-exchange reaction is determined by eq. (6). The isospin difference of the real parts of the amplitudes ${ }^{33} \mathrm{P}_{1}-{ }^{13} \mathrm{P}_{1}$ is plotted in fig. 4d. The integration from $2 \mathrm{fm}$ to infinity gives the same value as the integration from zero to infinity in this case, because the contributions below $2 \mathrm{fm}$ cancel. The charge-exchange reaction is sensitive to the region up to $4 \mathrm{fm}$, in sharp contrast to the annihilation.

In earlier experiments $[15,16]$ on the charge-exchange reaction, a dip between the forward peak and the large-angle fall-off was reported. It was interpreted as an interference between the scattering amplitude due to onepion exchange and that due to short-range interactions [15, 16]. The present analysis shows, on the contrary, that the two components in the differential cross-section both originate from one-pion exchange: the forward peak comes from the spin-spin term $v_{s s}$ and the large-angle part comes from the tensor term $\mathrm{V}_{\mathrm{T}}$ of OPEP, as demonstrated in fig. 5 using the present model. 
The elastic scattering is determined in eq. (5). In the elastic scattering, the long-range parts of the scattering amplitudes are mostly cancelled because they have opposite signs for $I=0$ and $I=1$ as shown in $\mathrm{fig}$. $4 \mathrm{c}$. We test the contribution of the long-range one-pion exchange by switching off the OPEP. It reduces the annihilation cross-section by $8 \%$ and the angle-integrated elastic cross-section by $13 \%$, at $290 \mathrm{MeV} / \mathrm{c}$. The charge-exchange cross-section becomes zero. The elastic cross-section is, therefore, mostly determined by mediumand short-range interactions.

\section{COMPARISON WITH THE OTHER MODEL}

Let us compare the present analysis with that where the Dover-Richard model is used [5]. Calculations with the Dover-Richard model [5] are shown in fig. 2. The potential in the Dover-Richard model consists of one-pion exchange, two-pion exchange, w exchange, and a complex annihilation potential of the Woods-Saxon type. The difference between the present model and the Dover-Richard model is only in the contribution of the two-pion and $w$ exchanges. When we switch off the two-pion and $w$ exchanges in the DoverRichard model, we do not find significant changes of the cross-sections except that the real-to-imaginary ratio of the forward elastic scattering amplitude decreases by about 0.1 .

Next, we switch off only the OPEP, in the Dover-Richard model, in order to test the contribution of long-range one-pion exchange, as was done in the previous section. The annihilation cross-section decreases by $9 \%$, the elastic cross-section by $16 \%$, and the charge-exchange cross-section by a factor of 100 at $290 \mathrm{MeV} / \mathrm{c}$. The OPEP mostly affects the charge-exchange reaction, in good agreement with the case in the present model.

In the previous sections, we have used eq. (1) in evaluating the range of interactions. It is justified because, as we have shown above, the twopion and w exchanges do not play major roles in determining the cross-sections

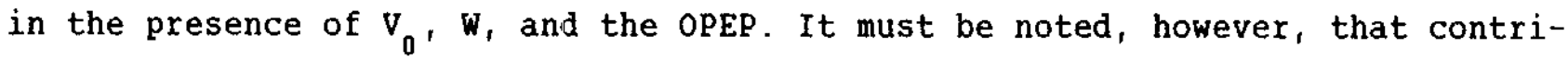
butions from short-range boson exchanges could be included in the real part of the annihilation potential, $v_{0}$.

\section{CONCLUSIONS}

We have shown that the antiproton-proton cross-sections at small momenta axe reproduced with a potential model which consists of a one-pion-exchange potential and a short-range annihilation potential. We have evaluated the ranges of the interactions, and shown a sharp contrast between the annihilation and the charge-exchange reaction: the annihilation takes place at short distances of less than $2 \mathrm{fm}$, while the charge-exchange reaction is sensitive to the region up to $4 \mathrm{fm}$. 
The range of the annihilation is characterized by the 'annihilation radius', which is around $1 \mathrm{fm}$. Since the annihilation cannot be mediated by the boson exchange, this result would mean that at a distance of $1 \mathrm{fm}$ the boson exchange is already not the dominant mechanism in the $\bar{N} N$ interaction. As the annihilation probability is distributed over the distance where the NN potential has a strong attraction, one is inclined to ask whether the boson exchange is a real mechanism of the nuclear attraction. In fact, finding out the exact roles of the two-pion and the w exchanges in the $\bar{N} N$ interaction is a crucial test of the boson-exchange model. Polarization measurements would be sensitive to those boson exchanges. The charge-exchange cross-sections could also provide information. In the present analysis, the fit of the chargeexchange cross-section at $290 \mathrm{MeV} / \mathrm{c}$ is poorer than that at $590 \mathrm{MeV} / \mathrm{c}$. This suggests contributions from other terms than those in eq. (1) at lower momenta.

\section{Acknowledgements}

The author wishes to thank B. Povh and Th. Walcher for stimulating discussions. He also thanks H.J. Pirner and J.-M. Richard for clarifying comments. 


\section{REFERENCES}

[1] พ. Brückner et al., phys. Lett. 158B (1985) 180.

[2] w. Brückner et al., Phys. Lett. 166B (1986) 113.

[3] W. Brückner et al., Phys. Lett. 169B (1986) 302.

[4] R.A. Bryan and R.J.N. Phillips, Nucl. Phys. B5 (1968) 201.

[5] C. Dover and J.-M. Richard, Phys. Rev. C21 (1980) 1466;

C. Dover, private communication (1984).

[6] J. Côté et al., Phys. Rev. Lett. $\underline{48}$ (1982) 1319;

M. Lacombe, private communication (1984).

[7] P.H. Timmers, W.A. van der Sanden and J.J. de Swart, Phys. Rev. D29 (1984) 1928.

[8] 0.D. Dalkarov and F. Myhrer, Nuovo Cimento 40A (1977) 152.

[9] A.M. Green and J.A. Niskanen, Nucl. Phys. A412 (1984) 448.

[10] M. Maruyama and T. Ueda, Prog. Theor. Phys. 74 (1985) 526.

[11] S. Furui and A. Faessler, Nucl. Phys. A424 (1984) 525.

[12] B. Povh and Th. Walcher, Comments Nucl. Part. Phys. 16 (1986) 85.

[13] H.P. Stapp, T.J. Ypsilantis and N. Metropolis, Phys. Rev. 105 (1957) 302.

[14] L.I. Schiff, Quantum mechanics (McGraw-Hill, New York, 1968), 3rd edn.

[15] M. Bogdanski et al., Phys. Lett. $\underline{62 B}$ (1976) 117.

[16] K. Nakamura et al., Phys. Rev. Lett. $\underline{53}$ (1984) 885. 


\section{Fiqure captions}

Fig. 1 The different terms of the potential in eq. (1).

Fig. 2 Elastic cross-sections at (a) $505 \mathrm{MeV} / \mathrm{c}$, (b) $287 \mathrm{MeV} / \mathrm{c}$, (c) $181 \mathrm{MeV} / \mathrm{c}$, and charge-exchange cross-sections at (d) $590 \mathrm{MeV} / \mathrm{c}$ and (e) $287 \mathrm{MeV} / \mathrm{c}$. The solid curves show the present calculation. The dotted curves show the calculation with the Dover-Richard model [5]. The dashed curves show the calculation with the Paris model [6]. The closed circles are experimental data from refs. [2] and [3]. The triangles are from ref. [16].

Fig. 3 The Argand diagram as a function of the beam momentum for ${ }^{33} \mathrm{p}_{1}$ and ${ }^{13} \mathrm{P}_{1}$ calculated with the potential in eq. (1). The arrow indicates the isospin difference at $290 \mathrm{MeV} / \mathrm{c}$. The dotted curve indicates the phase shifts when the OPEP is switched off.

Fig. 4 a) The incident plane wave, and the real and the imaginary part of the wave function of the solution of the Schrödinger equation, $\mathrm{R}_{1} \cdot \mathrm{kr}$, for ${ }^{13} \mathrm{P}$, at $290 \mathrm{MeV} / \mathrm{c}$. The imaginary potential $\mathrm{W}$ is also shown. b) The squared wave function $\left|R_{1}\right|^{2} \cdot(k r)^{2}$ and the annihilation probability $-W\left|R_{1}\right|^{2} \cdot(k r)^{2}$, for ${ }^{13} P_{1} \cdot$ c) The real part of $-j_{1} \vee R_{1} \cdot(k r)^{2}$ for ${ }^{33} \mathrm{P}_{1}$ and ${ }^{13} \mathrm{P}_{1}$. d) The difference, ${ }^{33} \mathrm{P}_{1}-{ }^{13} \mathrm{P}_{1}$, of the two curves in (c).

Fig. 5 The charge-exchange cross-sections at $590 \mathrm{MeV} / \mathrm{c}$ calculated with eq. (1) are shown by the solid curve. The dashed curve shows the calculation without $\mathrm{V}_{\mathrm{T}}$ of OPEP, and the dotted curve shows the calculation without $\mathrm{v}_{\mathrm{ss}}$. The closed circles are from ref. [3], the triangles from ref. [16]. 


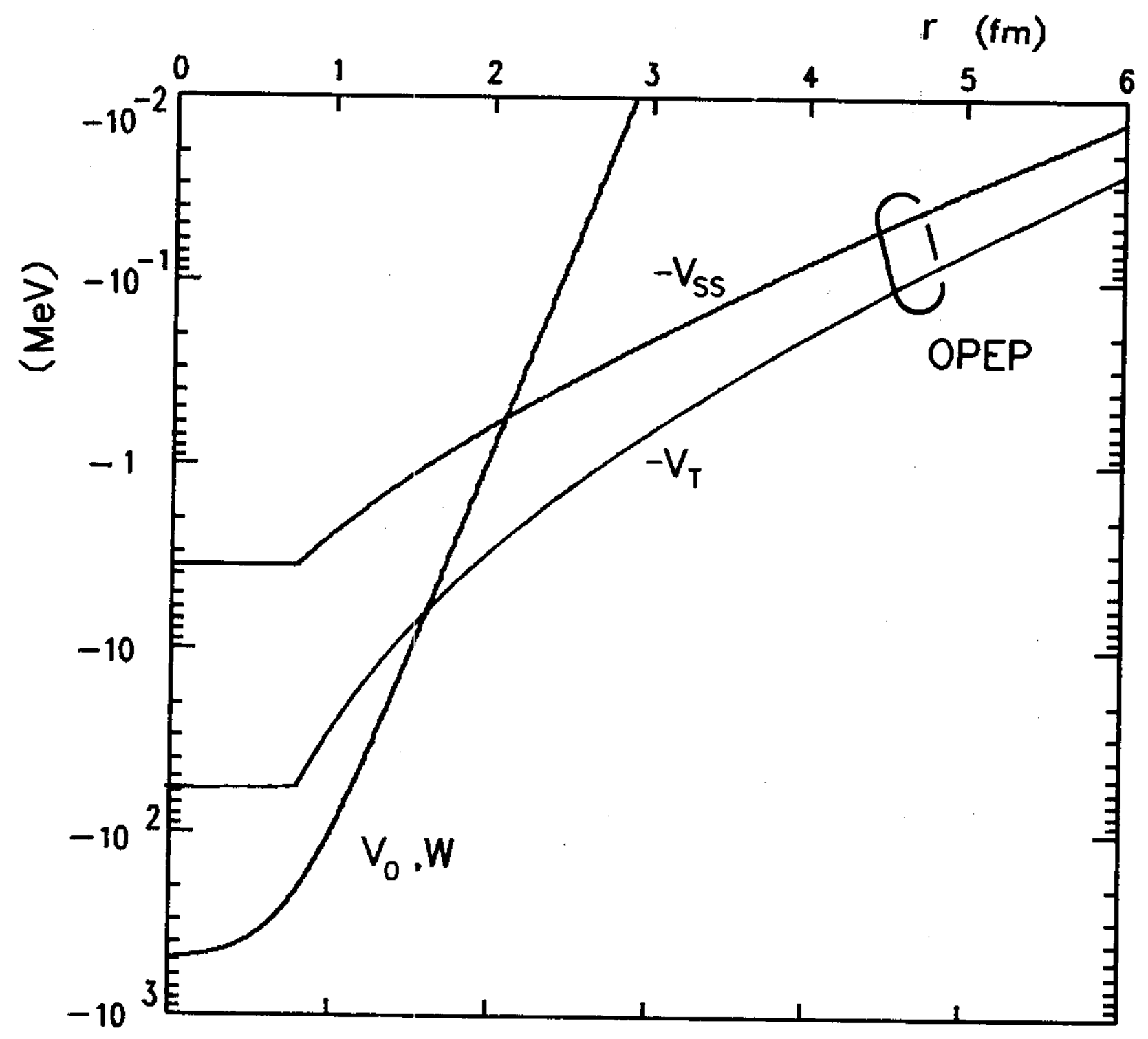

Fig. 1 


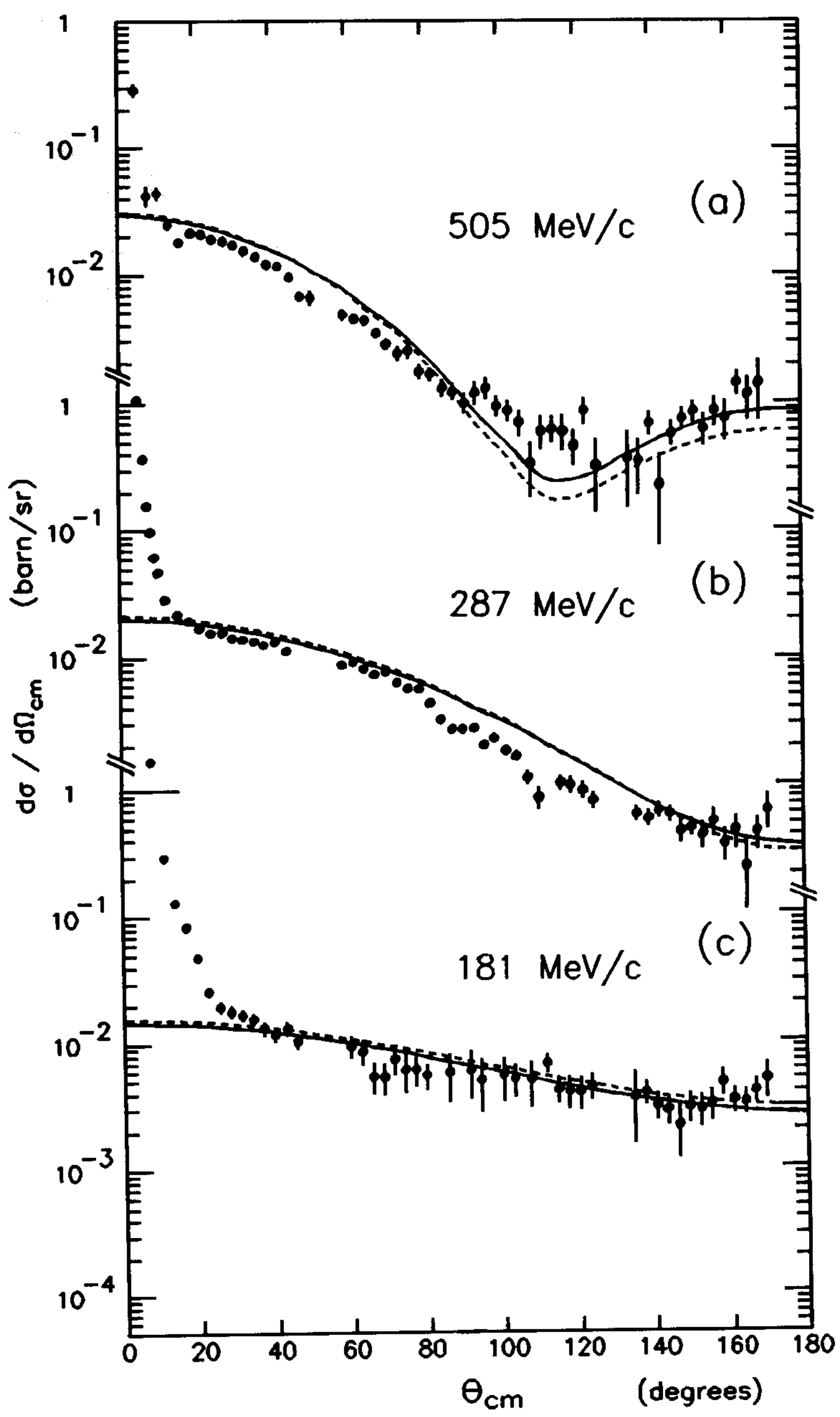

Fig. 2 


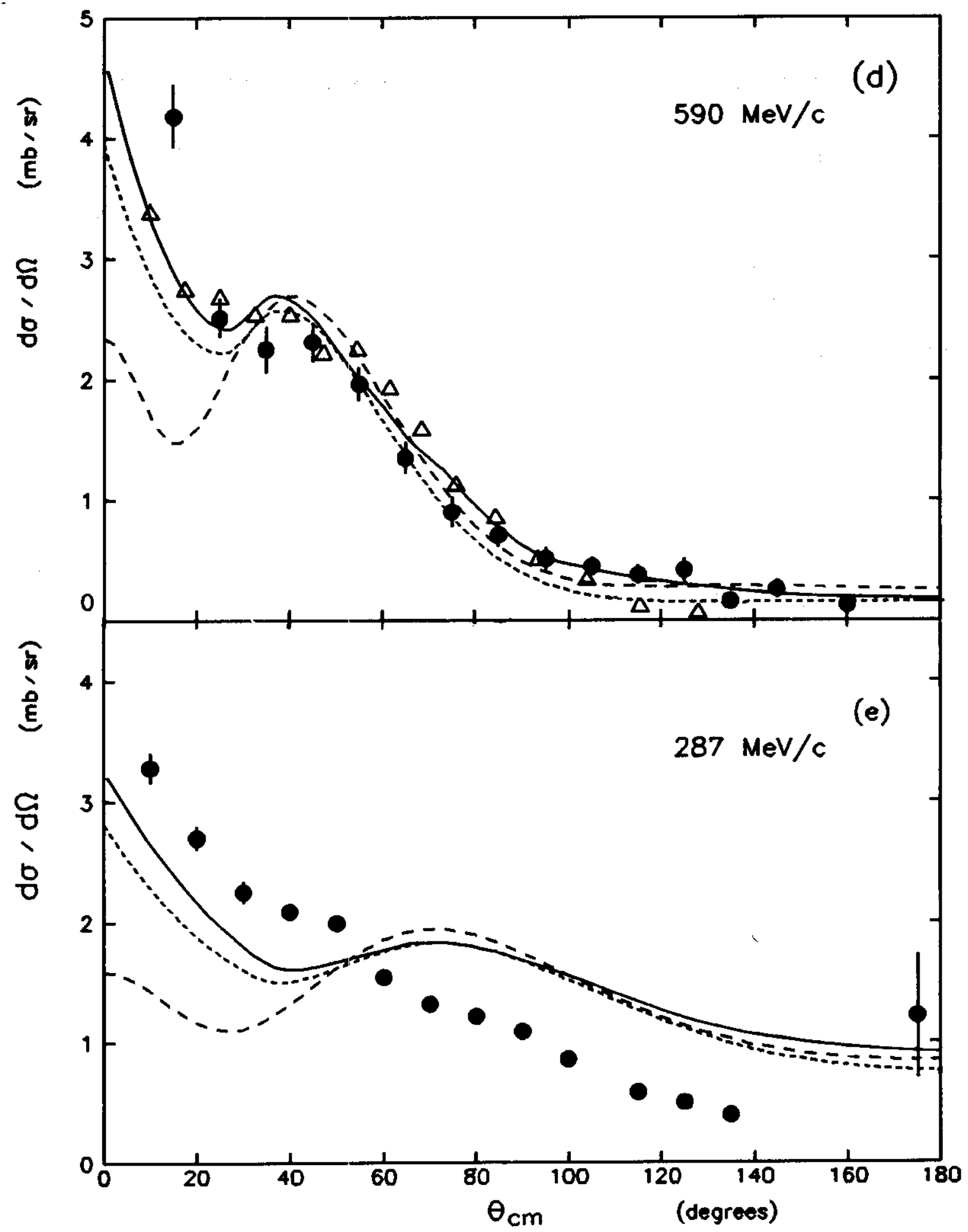

Fig. 2 


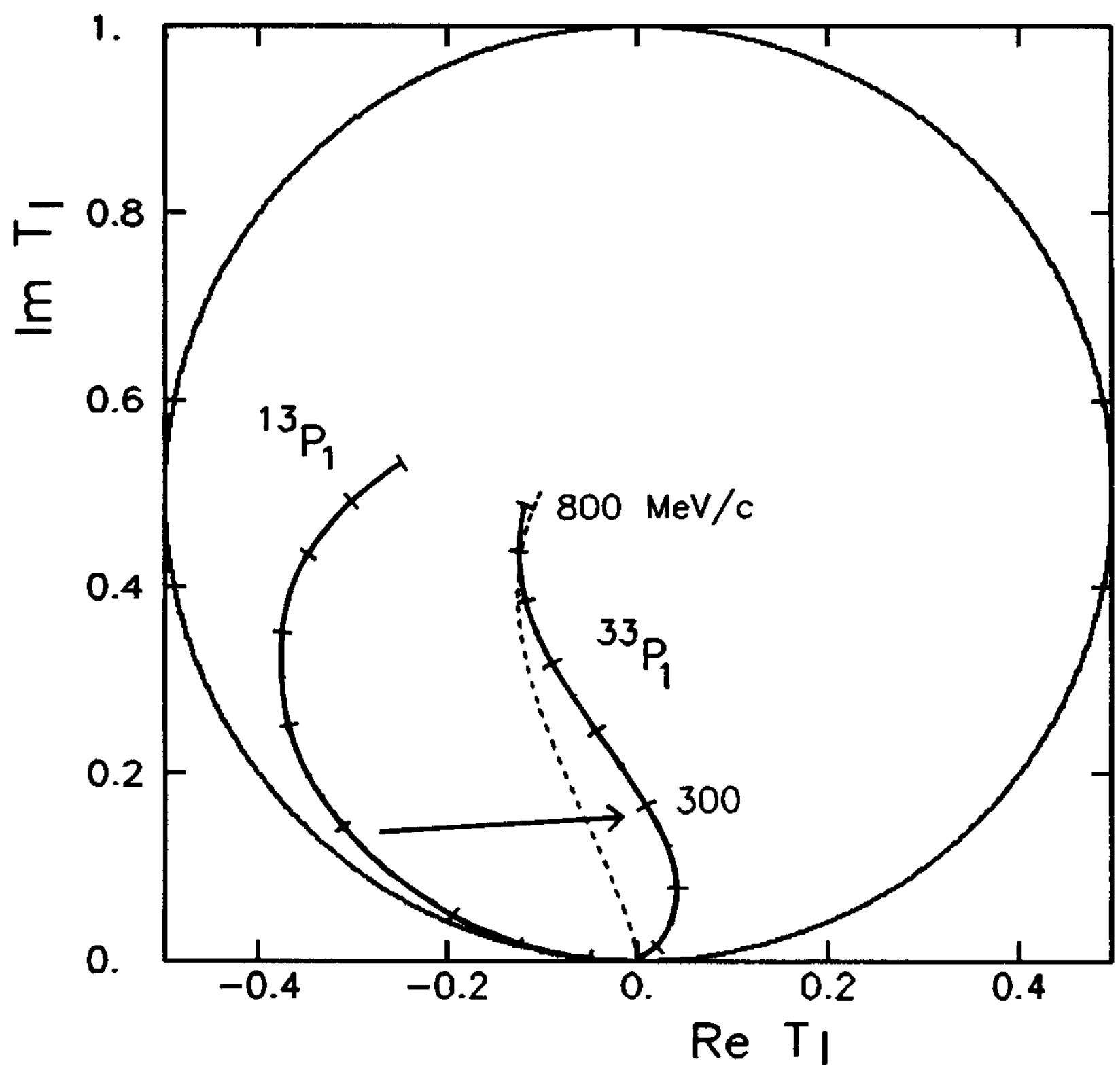

Fig. 3 


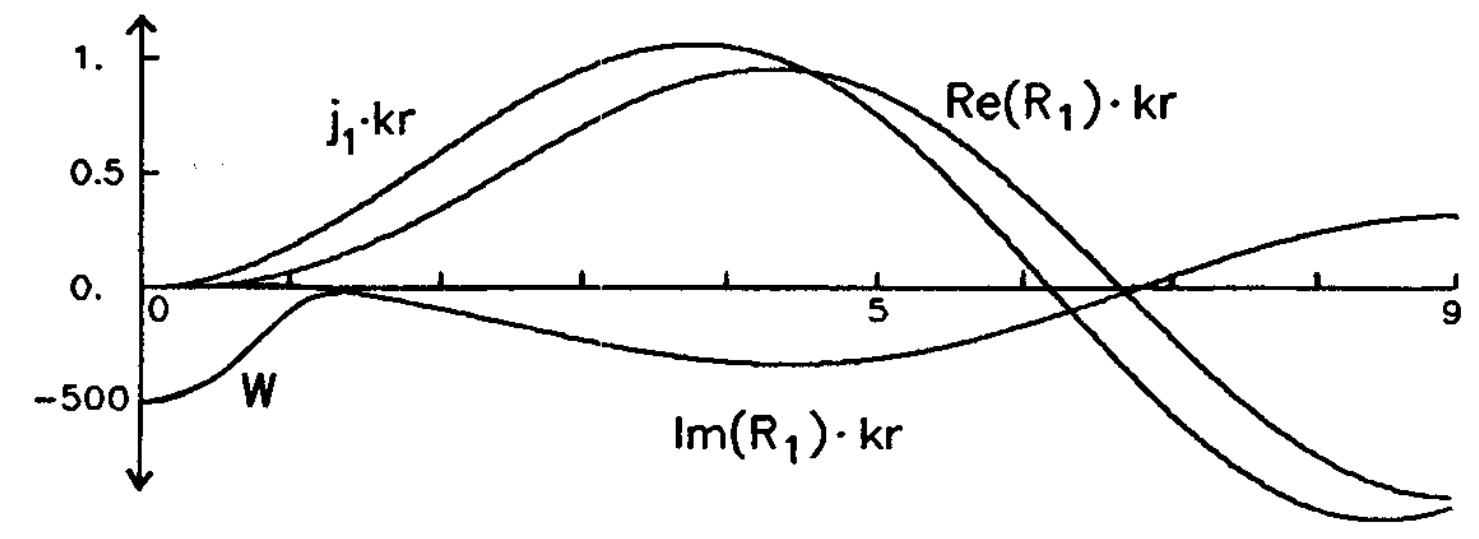

a)

产
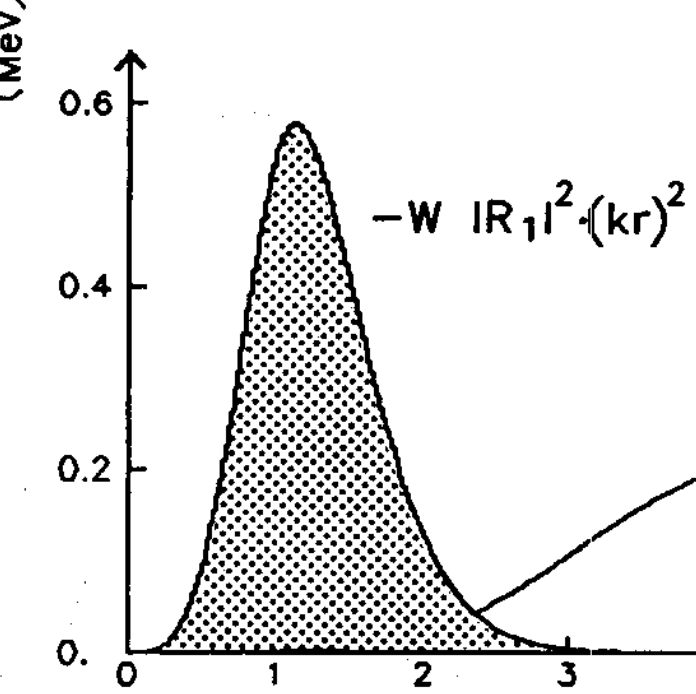

o.
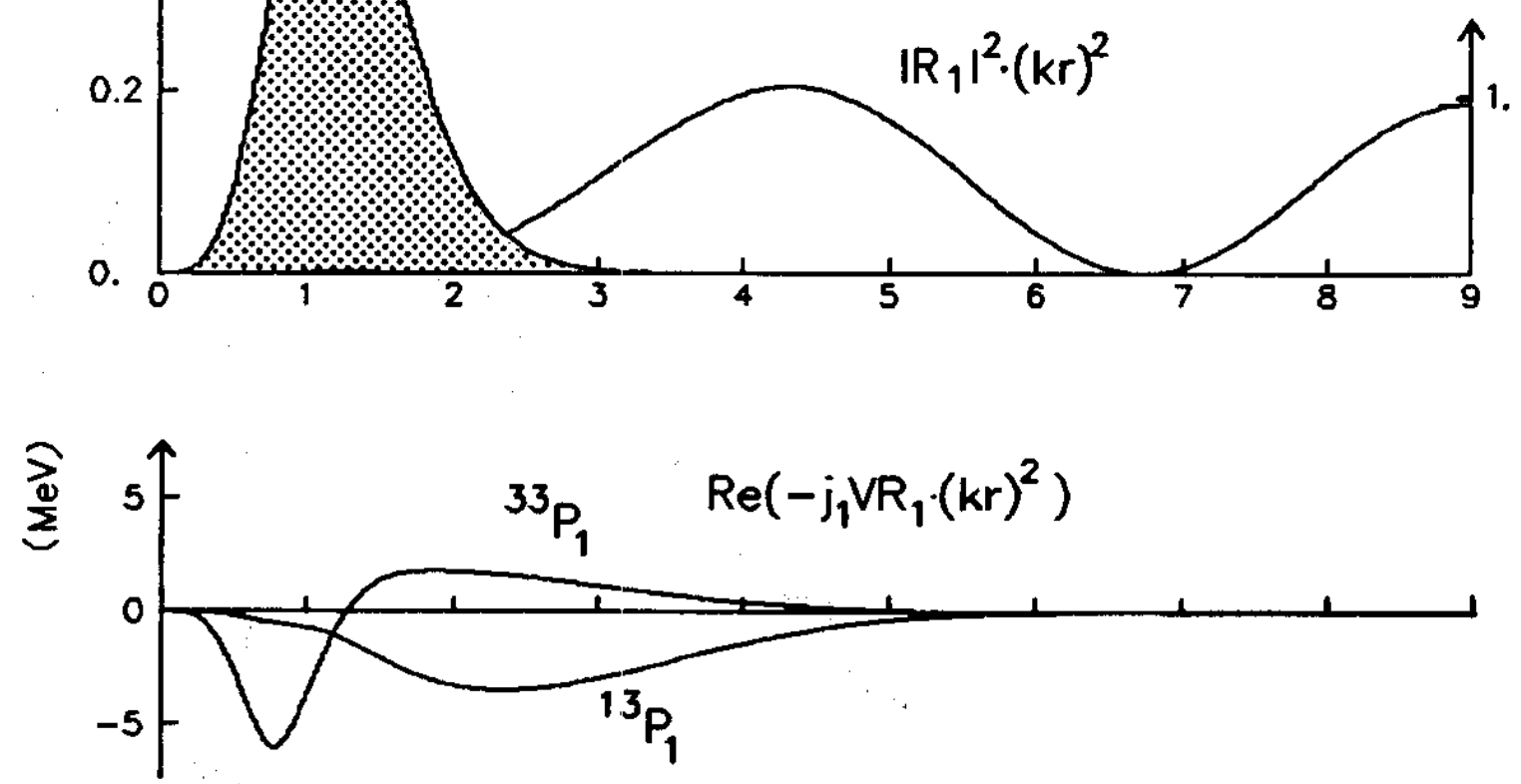

c)

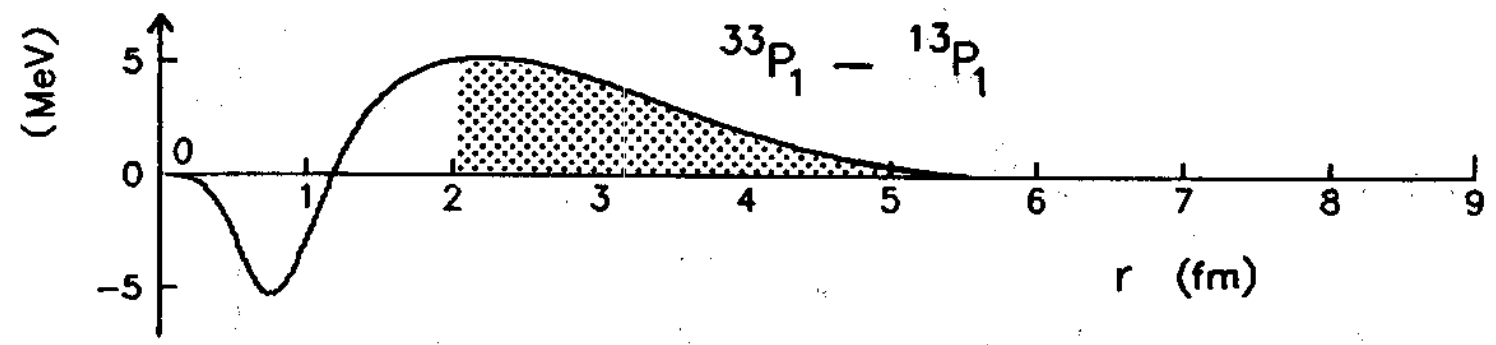

b)

d)

Fig. 4 


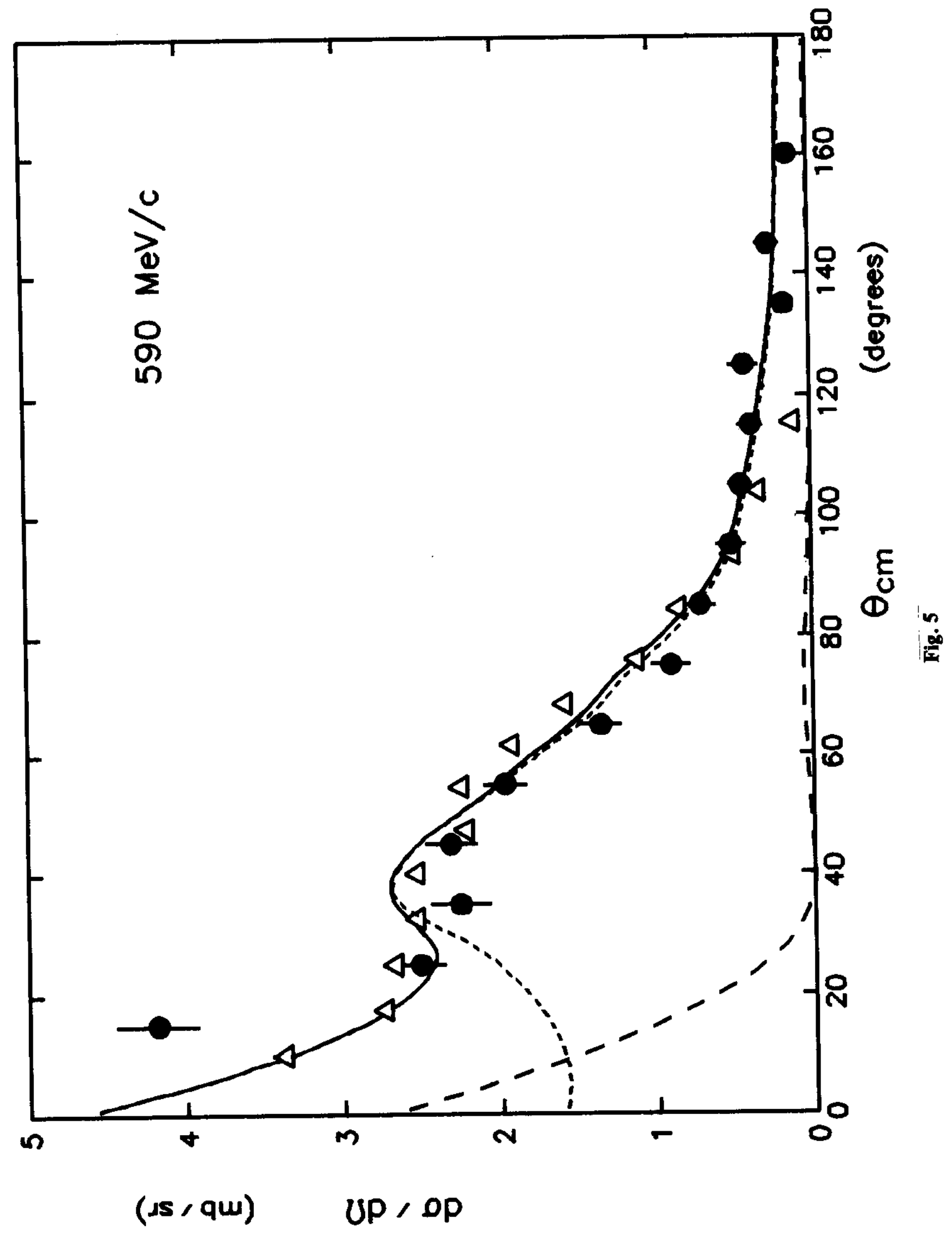

